\title{
The illusion of transparency and chromatic subjective contours
}

\author{
GLENN E. MEYER and MARY SENECAL \\ Lewis and Clark College, Portland, Oregon
}

\begin{abstract}
Subjective contours can be produced that include an illusion of edge and an extension of color throughout the area of the illusion. The phenomenological appearance is of a transparent colored shape in front of the background. Two explanations of this illusion are proposed. The first is that there is an assimilation of color analogous to brightness assimilation. The second is a variant of the stratification of depth theory of subjective contours. In it, the pattern elements lead to the illusion of a surface in front of the pattern elements. We thus predicted that an illusion of transparency would enhance the subjective contour. Metelli's model of transparency was used to quantify our prediction, and it was found that the possibility of transparency was a powerful predictor of the chromatic subjective contour.
\end{abstract}

Over the past decade, there has been a geometric expansion of research dealing with subjective contours. Most research, however, has been done with achromatic stimuli such as the now familiar Kanizsa triangle (Kanizsa, 1976, p. 48). This figure is typical of a subjective contour display and has several interesting characteristics. The illusory triangle is brighter (or darker) than its background, has sharp edges, is opaque, and seems to be in front of the background. Many studies have concentrated on the causes of this illusion, the phenomenological appearance of the contour and its extended area of brightness (or darkness if contrast was reversed), and the functional properties of the illusory edge (see Halpern, 1981, Kanizsa, 1976, 1979, Meyer \& Garges, 1979, for typical examples).

Relatively neglected has been the role of color in subjective contours. Several studies have indirectly dealt with it. Smith and Over (1976) produced a subjective-contour tilt aftereffect that was color-specific. Meyer and Phillips (1980) demonstrated that subjective edges can be enhanced by the McCollough effect and eliminated by a perceptual reorganization of stimuli. Both studies suggest that color information can be part of a subjective edge. Several studies have constructed Kanizsa-type displays using components matched in brightness but varying in color and have found much weaker subjective contours (Brigner \& Gallagher, 1974; Brussell, Stober, \& Bodinger, 1977; Frisby \& Clatworthy, 1975; Gregory, 1977). However, only three recent studies have looked at the pro-

Parts of these data were presented at the 1980 meeting of the Psychonomic Society, St. Louis, Missouri. This research was supported by Lewis and Clark College faculty research grants. The authors wish to thank Thomas Schoenaman and the anonymous reviewers for their helpful suggestions. Requests for reprints should be sent to: Glenn E. Meyer, Department of Psychology, Lewis and Clark College, Portland, Oregon 97219. duction of a subjective edge that is colored and bounds an area of extended illusory color. Both Varin (1971) and Ware (1980) found that a colored Kanizsa triangle is possible if the "wedge" cut into the black circles is filled with color. A subjective colored triangle of the same color is then visible. Van Tuijl (1975) reported a neon illusion that seems similar but uses lattices rather than shapes.

This paper deals with factors involved in the chromatic subjective contour. In current explanations of the brightness subjective contours, two camps are evident. The first is physiologically oriented and looks to edge detectors, Fourier analysis, retinal ganglion cells, brightness contrast, and assimilation as the crucial processes. Cognitive theories, on the other hand; propose depth cues, good figure, and analyses based on attention or problem solving strategies as the sources of these illusions. Total separation of these factors is not possible. However, both Halpern (1981) and Van Tuijl and Leeuwenberg (1982) have demonstrated that both factors interact in the formation of subjective contours.

How do these theories apply to the colored subjective triangle? Simple color contrast does not seem to be a factor. If a Kanizsa figure is made of red components (red solid circles with a piece cut out), a green subjective triangle is not reported, as would be predicted by a color contrast explanation. To get color extension, Ware and Varin found that the wedges have to be filled in with a color, and that it is this color that extends and not a contrasting color. Thus, there is a fundamental difference in appearance between a brightness subjective triangle and a color subjective triangle. The first seems opaque and obscures the background black circles by interposition. The color triangle seems transparent and overlaps the background circles. Achromatic versions of this transparent subjective contour exist as pro- 
duced by Kanizsa $(1976,1979)$. An example is seen in Figure 1D.

Our analysis of the illusion will center on the role of transparency. First, it is necessary to examine illusory transparency. It is illusory in the sense that a printed example has only one physical depth plane; there cannot be a transparent surface in front of the background surface. The transparency illusion has a fairly long history. It was well known to the Gestaltists (Ellis, 1967) and in the art world (Albers, 1963). Recently, Metelli $(1970,1974)$ has produced an algebraic theory of transparency based on Talbot's law of color fusion. An example of a transparent pear is seen in Figure 1A. A schematic of the four areas of varying grays is also presented (Figure 1C). Each letter represents the reflectance of its area. Metelli assumes that a color scission occurs. This means that the color of the grays (the paper itself) that compose the halves of the transparent object is perceptually split. Some is assumed to belong to the background. Some belongs to the objects. This occurs with area $p$ (Figure 1C) and with area $q$. This scission produces the perception of a transparent overlying shape. Metelli derives an index of transparency $(\alpha)$ which represents the proportion of color going to the underlying opaque surfaces (the backgound), $\alpha$ is calculated as follows: $\alpha=(p-q) /(a-b)$. Values range from 0 to 1 . A value of 0 would indicate that the pear would be seen as opaque and not transparent. A 1 would produce a completely transparent, and hence invisible, figure. A value of .5 produces the best illusion of a visible transparent shape.

Given the similarity of appearance of Metelli's transparency illusion and Kanizsa's transparent subjective contour (Figures 1A and 1D), it seemed to us that conditions conducive to transparency would be conducive to producing a chromatic subjective contour and color extension such that the illusion would be a transparent colored shape overlapping the dots and background. Ware (1980), on the other hand, discounted the role of transparency and suggested that assimilation was most important in the filling of the color. However, Kanizsa (1979) felt that assimilation was not a factor in the achromatic transparent subjective contour (Figure 9.13, $a$ and $b$, pp. 168-169). It is possible that both factors interact.

To test the predicted importance of transparency, it was necessary to apply Metelli's model to a subjective contour display similar to Kanizsa's and Varin's. In our displays, the circles with wedges removed (designated as "ears") and background were achromatic but varying in reflectance. The wedges or gray areas in Kanizsa's display ("corners") were colored. We predicted that the color would fill the rectangle and establish a sharp subjective contour between the elements of the pattern. The problem is to measure transparency in this situation. Metelli's equation for $\alpha$ is based on four reflectances: $a, b, p$,

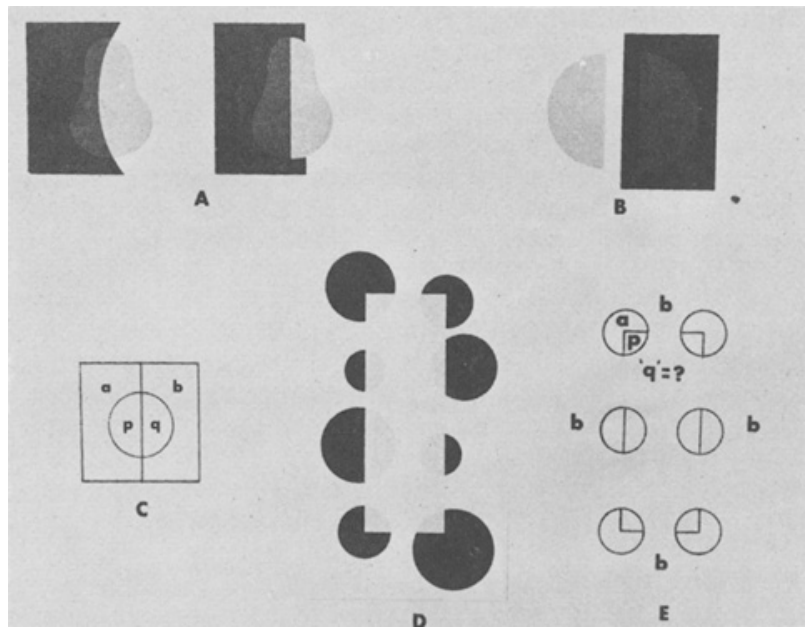

Figure 1. (A) An example of the transparency illusion producing a translucent pear and the elimination of the llusion with contonr misalignment. (B) An illustration that a surrounded figure produces no transparency. Overiap of the figure is necessary. (C) A schematic of Metelli's (1970, 1974) model of transparency. Each letter represents the reflectance of the paper cutout used to construct the figure. (D) Kanizas's (1976, 1979) subjective contour configuration producing both a subjective contour and transparency. (E) An application of Metelli's model to Kaniasa's illuslon. The reflectances of $a, b$, and $p$ are fixed; " $q$ " is unknown in the perceptud situntion as it is the area of illusion.

and $q$. In a subjective contour (see Figure 1E), $q$ is missing, because it is the area of illusion, but $a, b$, and $p$ are known. However, given $a, p$, and $b$, it is possible to calculate $\alpha$ as $q$ varies from $0 \%$ to $100 \%$ reflectance. In a Metelli display, this should indicate the strength of the transparency illusion. We calculated two indices for the subjective contour displays: first, the percent of configurations, given a fixed a, $p$, and $b$, for which transparency would be possible ( $a$ between 0 and 1 ) as $q$ varied from 0 to 100; and second, the strength of the transparency illusion for the possible values as though it were a complete Metelli figure.

Subjects rated the strength of the subjective chromatic contour as we varied the reflectances of a (ear) and $b$ (background). The reflectance of colored area $\mathrm{p}$ was fixed. We predicted that if transparency was possible given these values in a Metelli display, then there would be a strong illusion of a subjective colored rectangle.

\section{METHOD}

\section{Subjects}

Ten undergraduate psychology majors acted as observers. All had normal or corrected acuity and were naive to the purpose of the study.

\section{Stimull}

A rectangular configuration was used to produce a subjective contour on a $3 \times 5$ in. Munsell matte-finish swatch. A hypothetical 
rectangle of $2.5 \times 4.5 \mathrm{~cm}$ was used as a starting point. At each corner of the rectangle a $1.9-\mathrm{cm}(3 / 4-\mathrm{in}$.) circle was mounted, with its center at the corner of the rectangle. Two more circles were mounted in between the corner circles along the long side of the rectangle. These circles were bisected by the side of the rectangle (see Figure 1E for a schematic).

The stimuli varied in the reflectances of the background and the ears. Eight Munsell swatches varied the reflectance of the background (N2-3.12\%, N3-6.5\%, N4-12\%, N5-19.77\%, N6$30.05 \%, \mathrm{~N} 7-43.06 \%$, N8-59.1\%, N9-78.66\%). Two black and white ears were made from Avery black-and-white 3/4-in.-diam press-on labels (reflectances, $14.5 \%$ and $88.9 \%$, respectively). Gray ears of same diameter were cut from Munsell N6 swatches. The colored corner was a Dennison radiant orange 3/4-in. presson label (76.2\% reflectance). Reflectance values were either those of the Munsell standards or measured using a Photo Research Spectra Spot Meter Model UB $14^{\circ}$ and a RS-1 reflectance standard. Thus, there were 24 stimuli ( 8 backgrounds $\times 3$ ears).

\section{Procedure}

The rating method used to determine strength of the subjective contour emphasized the appearance of the color extension. First, subjects were shown the achromatic Kanizsa pattern which produces the illusion of a translucent gray rectangle. All subjects could see this illusion. Next, the subjects were shown the color display using the N3 background and white ear. Pilot data had indicated that this produced a strong color extension in the subjective rectangle. The subjects were told to rate an orange appearance of this strength as a 10 . Next, they were shown an N9 background, black ear display and told they would see similar displays with varying ears and backgrounds and would have to rate the strength of the illusory orange as compared with the standard 10. Any questions were answered. The swatches were placed individually on a table, at approximately $40 \mathrm{~cm}$ in front of the subject, under fluorescent lighting (\$35.2 1x illumination). Since there were 24 displays, each subject received a different random order. There was no time limit to report, but subjects made the ratings in a few seconds.

\section{RESULTS}

Two analyses were conducted. The first was an analysis of variance on the rating data. The second used regression techniques to compare these data to predictions made from Metelli's model.

The rating data are presented in Figure 2. The two factors in the analysis are ear and background reflectance. The main effect of ear was significant $[F(2,207)=22.45, p<.001]$. The main effect of background was not significant $[F(7,207)=1.14$, $p>.05$ ] due to the significant ear $\times$ background interaction $[F(14,207)=2.67, p<.05]$.

The second analysis attempted to match our data to a prediction from Metelli's model. As mentioned earlier, we used the following procedure. First, we calculated the percentage of the time transparency would be possible ( $\alpha$ between 0 and 1) as q (the missing area in the subjective display) varied from $0 \%$ to $100 \%$ reflectance in a typical Metelli display. This calculation reflects the constraints of Metelli's equation such that $a-b$ and $p-q$ have the same sign and $|a-b|>|p-q|$. Next that we calculated two measures of transparency strength if the value of $q$ allowed transparency: (1) the mean of the possible transparency values, and (2) the absolute deviation

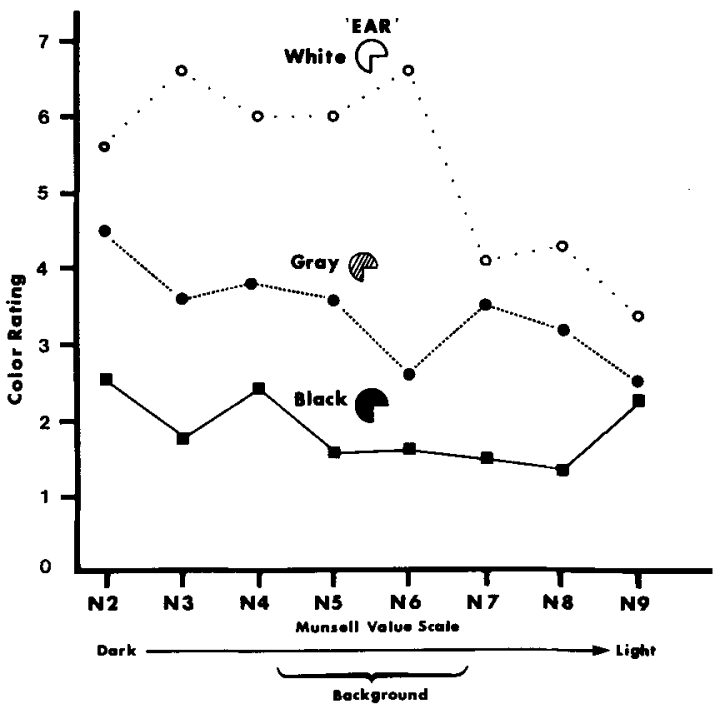

Figure 2. Mean ratings of the illusory color strength. The abscissa represents the Munsell value of the background of our figures (area b in Figure 1E). "Ear" represents the appearance of aren $p$ (Figure 1E) in our figure. Reflectances for these components are given in the method sections.

of the mean transparency value from .5. Since Metelli suggests maximal transparency illusion at $\alpha=.5$, we thought deviation from this value might predict a weakened illusion. Figure 3 presents the percentage of time transparency is possible for our background and ear reflectances. Figure 4 represents the absolute deviations similarly computed. We suggested that a high percentage of configurations with possible transparency values and a strong measure of

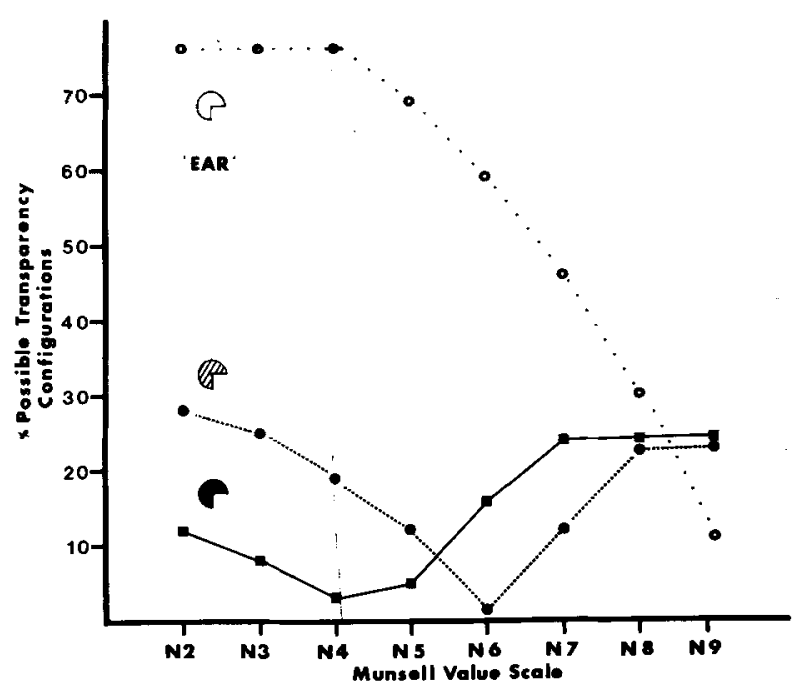

Figure 3. Percent of configurations for which tranoparency is possible ( $a$ between 0 and 1 ). This was computed with $a, b$, and $p$ fixed and q varying between $0 \%$ and $100 \%$ reflectance. Abscissa represents the Munsell value scale of area b (background). "Ear" represents area $\mathbf{p .}$ 


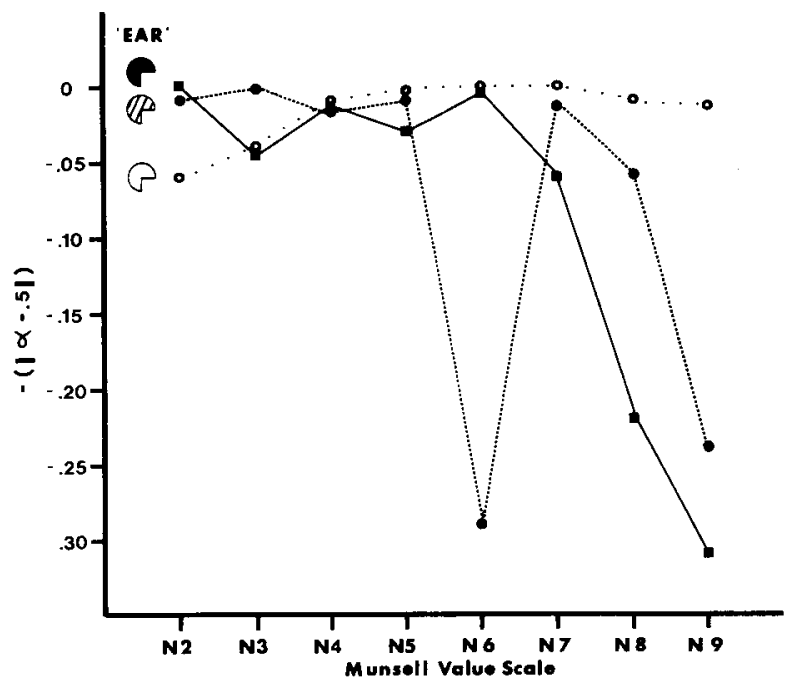

Figure 4. The abscissa and "ear"' are as in Figures 2 and 3. First, the mexn transparency $(a)$ of the possible configurations (computed as in Figure 3) for the 24 combinations of background and ear were computed. This value was subtracted from .5 , which is the best value for the transparency illusion (see text) and the absolute value plotted. This assumed that a deviation from .5 would reduce the illusion.

transparency would predict a strong illusion. A stepwise multiple regression tested the fit of the data to these three predictors. The multiple $R$ of .86 was highly significant $[F(2,21)=29.54, p<.001]$. However, only the percentage of possible transparent configurations was a significant predictor as it explained $64.2 \%$ of the variance $[F(1,21)=51.45$, $\mathrm{p}<.001]$. The average transparency value and the deviation score were not significant predictors $(p>$ $.05)$, contributing less than $10 \%$ of the variance.

\section{DISCUSSION}

Our data provided a surprisingly good fit between the possible number of transparent configurations in a Metelli display and the rated strength of the illusory color in the subjective contour display. Our $\mathrm{R}$ was .86 with percentage of possible configurations predicting $64.2 \%$ of the variance. In comparison, Halpern (1981), using a multiple regression approach to relate Kanizsa type rectangles variance to depth, brightness contrast, and figural cues, predicted $14 \%$ of the variance. It was disappointing, however, that our measures of transparency strength did not contribute. Interestingly, our deviation score estimate of transparency strength (Figure 4) predicts the main effect of "ear" in our data. The smallest absolute deviation from a perfect illusion of transparency $(\alpha=.5)$ is found for the white ear $(-.01)$; for the gray, it is -.08 , and for the black, -.09 . Despite this, the overall predictive use of transparency strength was minimal. Various weightings, transformations, and interactional combinations of percent possible configurations and the other variables did not improve prediction and will not be discussed further. Thus, despite the intuitive appeal of a measure of transparency strength, we conclude that the probability of transparency is more potent.

We believe that these data support an interactionist view of subjective contour formation. Perhaps Van Tuijl and Leeuwenberg (1982, p. 114) best exemplify this position with regard to Kanizsa-type figures when they state: "It is argued that (a) local brightness effects, caused by lateral inhibition are indeed necessary for the occurrence of subjective contours and that (b) the relative simplicity of the illusory interpretation of the pattern determines, ceteris paribus, the strength with which the phenomenon occurs." Thus, given the necessary physical parameters, cognitive factors (in their case, the efficiency of the interposition interpretation of the figure based on Leeuwenberg's coding theory) can determine subjective contour strength. They feel that "neither factor alone can explain all the facts known today about subjective contours"' (p. 114).

A similar analysis can be applied to our data. It is possible that a spread of color may occur through assimilation. This could act as a cue for possible transparency, and the probability of transparency would then have a dramatic effect on illusion strength. Several lines of evidence support this.

As previously reviewed, higher contrast strengthens subjective contours and might be a factor in predicting our illusion if it aided assimilation. We therefore calculated the contrasts of our stimuli comparing "ear" vs. background swatch and "ear" vs. colored corner. We entered these contrast values for each stimulus, with the deviation and transparency score, into a similar regression analysis, as described above. In both analyses, deviation and transparency were again nonsignificant. Contrast of ear vs. background was a significant predictor $(p<.05)$, but only explained $28.4 \%$ of the variance, compared with the $64.2 \%$ for our transparency probability measure. These proportions were significantly different $(p<$ $.05)$. If contrast of ear vs. background are both entered into the same regression, only transparency probability is a significant factor $(p<.01)$. Contrast is not $(\mathrm{p}>.25)$. The unique variance contribution of transparency was $64.2 \%$, while that of contrast was $0.34 \%$. Similarly, contrast of ear vs. swatch was not a powerful predictor. Note that this contrast would remain constant for the series of backgrounds for each ear. When entered into analyses as done above, it did not contribute significantly $(p>.10$ in all cases). Thus, transparency probability seems a potent predictor as compared with contrast.

The neon illusion, which produces a spread of color through a lattice with a phenomenological ap- 
pearance similar to that of our displays, shows properties in accord with our viewpoint. Van Tuijl and Leeuwenberg (1979) found that the structural information, as defined by coding theory, must be efficient for the neon illusion to occur. Their work, both with subjective contours and the neon illusion, support an interaction of low- and high-level factors. Thus, measures of structural property, such as coding theory's efficiency of interposition or our transparency probability, seem to tap the likelihood of a depth stratification or other separation of figural components in such a way as to suggest the existence of a colored plane or achromatic plane (their application of coding theory to Kanizsa figures; Van Tuijl \& Leeuwenberg, 1982). If there is a high likelihood, both chromatic and achromatic subjective contours are strong.

In our displays, it may be that there is an evaluation of the possible presence of an overlapping colored area which the configuration would seem to suggest (Kanizsa, 1979). Also, if assimilation of color is present, it might convert the $t$ junctions of the ear, corner, and background into $\mathrm{x}$ junctions (due to the color spread); these are powerful cues for transparency and overlap. Given that overlap and transparency are reasonable and efficient interpretations of the pattern, the chromatic subjective contour and color extension is dramatically heightened by the probability of transparency for a given set of structural elements. As a result, we get a configuration that maximizes a stratification in depth by an interaction of factors.

A simple demonstration suggests the importance of these interactions. Kanizsa (1979, Figure 9.13, $a$ and $b$ ) produced a transparent subjective contour similar to Figure 1d. He also replaced the gray hemicircles with gray strips that ran uninterruptedly along the sides of the illusory rectangle. Solid stripes would seem to be an even stronger stimulus for assimilation based solely on low-level factors, since there is more gray area. However, the illusion of the transparent overlapping gray square was eliminated. Kanizsa (p. 169) concluded that "the brightness transformation phenomena illustrated here should be considered as a consequence of the phenomenal presence of transparency and not its cause." Similarly, we took our best configuration (white ears on N3 background) and compared it to one in which orange strips replaced the orange hemicircles. The color extension was not apparent to us or to 10 naive subjects. These data are easy to interpret given an interactionist position. If assimilation is present from the strips, it cannot produce the information necessary for the evaluation of overlap (see Figure 1 for the necessity of overlap and alignment) because the hemicircles are no longer seen. Thus, the powerful strengthening of the illusion due to cognitive factors was not present. Thus, assimilation by itself cannot support the illusion, although it does act as a cue.

Ware (1980), however, concluded that transparency was not as important a factor as assimilation. His data, however, were not a powerful test of the transparency hypothesis. Ware constructed Kanizsa triangles using various ear and corner combinations of color and gray. In several patterns, transparency was not possible (red ear/green corner and vice versa, similar red-blue displays). Given that subtractive color mixture seems the rule in this type of transparency (Beck, 1975), the lightnesses and pigment preclude transparency. Three configurations were compatible with transparency (gray ear/blue, red, or green corner). Six subjects were shown each display.

For the red-green, red-blue combinations, Ware reported that 12 subjects saw a subjective triangle of the same color as the corner. Eleven reported no color, with one color report being anomalous. Thus, the ratio of successful "assimilation" vs. contrary reports was $1: 1$ in a configuration not compatible for transparency. In the gray ear/red, blue, and green corner conditions, 12 subjects reported a colored triangle of the same color as the corner and, 6 reported no color. These patterns are compatible with transparency and yield a 2:1 success ratio. Thus, when transparency is possible, Ware shows a large and significant increase $\left(\chi^{2}=4.01, \mathrm{p}<.05\right)$ in color contour report.

The same analysis can be applied to the other conditions. The green ear/blue corner condition would predict a yellow on a transparency basis, but it would have to spread outside the rectangle. This would imply that the entire circle was blue and that only its corner was unobscured by a transparent overlay. This configuration does not seem a likely one. Since some sense of "good figure" seems necessary for transparency (Figure 1, a and b) one might expect a weakened illusion. The ratio of color to no-color report is $1: 1$, again lower than the $2: 1$ ratio reported with the gray ear/color corner display.

Ware's strongest test of assimilation vs. transparency is a blue ear/green corner triangle. Assimilation predicts a green triangle. A subtractive mixture transparency model predicts a yellow color extension as if there was a yellow triangular piece of color filter overlapping the blue dots. Four subjects reported a green triangle. One reported a yellow, and one a yellow-green. Is this a good test of transparency? Unfortunately, it is not. First, there is a problem of configuration. It is well known (Fuchs, 1928, cited in Ellis, 1967) that to produce the color (Albers, 1963; Beck, 1975) transparency illusion, overlap of colored areas is necessary (see our Figure 1 , $a$ and $b$ ). For example, a green square completely surrounded by blue does not produce an im- 
pression of a yellow square over a solid blue. However, a blue circle and yellow circle intersecting, as in a Venn diagram, with the intersection green does produce the illusion of transparency. Obviously, looking just at the green intersection area only, as if through a reduction tube, yields green. Transparency needs overlap. It is thus clear that for color transparency to occur in displays of two colors, both colors have to be visible and there must be an area of overlap. In Ware's blue-ear/green-corner situation, there is obviously no yellow visible. And, for Ware, without the presence of both colors (blue and yellow) plus the green, it might be stretching the computational abilities of the visual system for it to split the green into blue and yellow, given that there was no yellow present. In our displays the reflectance parameters were conducive to transparency. The color that extended (orange) was the same as that seen on the overlapping area/orange ear. This might be an easy construction.

Thus, Ware's conclusive test is a weak configuration for transparency. It contains a high level of ambiguity. Despite this, two subjects reported some yellow. Ware discounts these subjects and feels they might have really seen desaturated green and confused it with yellow. However, assimilation is supported by the majority response. This is hard to justify with six subjects. If, by chance, three subjects saw yellow, rather than two, would transparency not be a factor? It is obvious that these numbers are too small to allow any conclusions to be drawn. In an ambiguous configuration, there may be assimilation (the four subjects saying "green") but an organization based on transparency was also reported (two subjects saying "yellow" or "yellow-green").

There is one last problem with Ware's data in light of our study. We constructed several red-ear/greencorner displays using Dennison red and green presson labels. On a white background (N9), the illusory area is clearly pink to the authors and large numbers ( 30) of naive subjects. On a dark background (N3), the rectangle was green. We are investigating these patterns further, but these findings are troublesome for Ware's conceptualization. The pink seems to indicate a contrast color, not assimilation. For the following reasons, it is not a simple case of contrast. First, a stimulus made of just green areas (just "corners" internal to the rectangle) produces no, or a very much weaker, pink. A rectangle outlined by just the red ears produces no color. Apparently, the red/ green border in the circles is necessary. These data contradict what Ware found with three subjects who reported a green triangle. Similar findings have been reported with the neon illusion, and a relation to the Ehrenstein illusion has been suggested (Van Tuijl, 1975).

Our red/green data does not address the issue of transparency, since the red/green corner and ear are difficult components for the necessary subtractive color mixture. We mention this because illusory contours are not simple and probably will have as many varieties as achromatic subjective contours.

\section{CONCLUSION}

To summarize and conclude, we think we have demonstrated that transparency is a factor in chromatic subjective contours. First, by extending Metelli's model to a configuration conducive to transparency, we found a good fit of model to subjects' rating of color strength. If cognitive factors aid in perceiving subjective contours, a depth stratification based on transparency seems to be one of them.

The transparency approach might be relevant to other illusions. The neon illusion has already been mentioned. White (1979, p. 413, Figure 1) presented an illusion of perceived lightness which looks like a translucent white rectangle superimposed over a grating. While White (1981) explains the illusion on the basis of contrast and assimilation, we applied our computer program to his reflectances (as measured from the journal figure) and found $61 \%$ possible transparent configurations. An inspection of Figure 3 shows that this would produce a strong illusion of a transparent subjective contour.

Transparency seems to be a potent factor in producing a subjective contour with both illusory edges and color extension. It is not the only factor. Van Tuijl (1975) also found color spread and contrast complementary colors possible in the neon illusion. It seems that many processes are active. Quantitative approaches offer the possibility of separating these factors and examining their interactions. As with brightness based subjective contours (Halpern, 1981; Van Tuijl \& Leeuwenberg, 1982), a range of high- and low-level structures is involved.

\section{REFERENCES}

Albens, J. Interaction of color. New Haven: Yale University Press, 1963.

BEck. J. The perception of surface color. Scientific American, $1975,233,62-75$.

Brigner, W. L., \& Gallagher, M. B. Subjective contours: Apparent depth or simultaneous brightness contrast. Perceptual and Motor Skills, 1974, 38, 1047-1053.

Brussell, E. M., Stober, S., \& Bodinger, D. M. Sensory information and subjective contour. American Journal of Psychology, 1977, 90, 145-156.

Ellis, W. D. A source book of gestalt psychology. New York: Humanities Press, 1967.

Frisey, J. P., \& Clatworthy, J. L. Illusory contours: Curious cases of simultaneous brightness contrast. Perception, 1975, 4, 349-357.

GREGORY, R. L. Vision with isoluminant colour contrast: 1. A projection technique and observations. Perception, 1977, 6, 113-119. 
Halpern, D. F. The determinants of illusory-contour perception. Perception, 1981, 10, 199-213.

Huruich, L. M. Color vision. Sunderland, Massachusetts: Sinauer Associates, 1981.

Kanizsa, G. Subjective contours. Scientific American, 1976, 234, 48-52.

KantzsA, G. Organization in vision. New York: Praeger, 1979.

Metelli, F. An algebraic development of the theory of perceptual transparency. Ergonomics, 1970, 13, 59-66.

Meteldi, F. The perception of transparency. Scientific American, $1974,230,90-98$.

Meyer, G. E., \& Garges, C. Subjective contours and the Poggendorff illusion. Perception \& Psychophysics, 1979, 26, 302304.

Meyer, G. E., \& Phillips, D. Faces, vases, subjective contours and the McCollough effect. Perception, 1980, 9, 603-606.

Smith, A. T., \& OVER, R. Color-selective tilt aftereffects with subjective contours. Perception \& Psychophysics, 1976, 20, 305-308.

VAN TuIJ, H. F. J. M. A new visual illusion: Neonlike color spreading and complementary color induction between subjective contours. Acta Psychologia, 1975, 39, 441-445.
Van TuiJl, H. F. J. M., \& Leeuwenbera, E. L. J. Neon color spreading and structural information measures. Perception \& Psychophysics, 1979, 25, 269-284.

Van TuiJl, H. F. J. M., \& Leeuwengerg, E. L. J. Peripheral and central determinants of subjective contour strength. In H. G. Geissler \& P. Petzold (Eds.), Psychological judgment and the process of perception. Berlin: VEB Deutschen Verlag der Wissenschaften, 1982.

Varin, D. Fenomini di contrasto e diffusione chromatic nell' organizzazione spaziale del campo percettivo. Rivista di Psicologia, 1971, 65, 101-128.

W Are, C. Coloured illusory triangle due to assimilation. Perception, 1980, 9, 103-107.

White, M. A new effect of pattern on perceived lightness. Perception, 1979, 8, 413-416.

WhITE, M. The effect of the nature of the surroundings on the perceived lightness of gray bars within square-wave test gratings. Perception, 1981, 10, 215-230.

(Manuscript received June 1, 1982;

revision accepted for publication March 7, 1983.) 